\title{
NF-kB as a therapeutic target in cancer
}

\section{Robert Z. Orlowski and Albert S. Baldwin,J r}

\begin{abstract}
The transcription factor nuclear factor (NF)-kB is activated in certain cancers and in response to chemotherapy and radiation. The transcriptional activation of genes associated with cell proliferation, angiogenesis, metastasis and suppression of apoptosis appears to lie at the heart of the ability of NF-kB to promote oncogenesis and cancer therapy resistance. Supporting these findings are recent experiments, performed in vitro and using xenograft models of cancer, which implicate NF-kB inhibition as an important new approach for the treatment of certain hematological malignancies and as an adjuvant approach in combination with chemotherapy or radiation for a variety of cancers. Clinical trials with drugs that block NF- $k B$ are currently in progress with promising results. However, as there is currently no drug that blocks specific NF-kB activation, conclusions drawn with small-molecule inhibitors must be interpreted carefully.
\end{abstract}

Published online: 1J uly 2002

First discovered and studied as a major activator of immune and inflammatory function via its ability to induce expression of genes encoding cytokines, cytokine receptors, and cell-adhesion molecules [1,2], the transcription factor nuclear factor (NF)- $\mathrm{kB}$ recently has been linked to control of cell growth and oncogenesis. The roles for NF-кB in cancer appear to be complex, but are likely to involve the ability of this transcription factor to control apoptosis and cell-cycle progression, and possibly cell differentiation, angiogenesis and cell migration. Importantly, it has been reported that NF- $\mathrm{KB}$ is activated in cancer cells by several chemotherapies and by radiation, and that in many cases this response inhibits the ability of the cancer therapy to induce cell death [3]. Here, we discuss the potential for the development of inhibitors of NF- $\kappa \mathrm{B}$ as primary as well as adjuvant approaches to cancer therapy.

\section{The NF-kB family and its regulation}

The five members of the NF- $\mathrm{BB}$ family are p50/p105

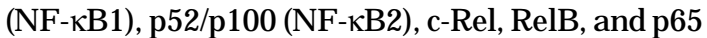
(RelA). These proteins are characterized by their Rel homology domains, which control DNA binding, dimerization and interactions with inhibitory factors known as IкB proteins (reviewed in Refs [1,2]). Three major forms of I $\mathrm{B}$ h have been identified, I $\mathrm{B} \alpha, \mathrm{I} \kappa \mathrm{B} \beta$, and $\mathrm{I} \kappa \mathrm{B} \varepsilon$, which have been generally shown to localize $\mathrm{NF}-\kappa \mathrm{B}$ proteins in the cytoplasm [1,2]. An unusual member of the I $\mathrm{KB}$ family is Bcl-3, which interacts with p50 and p52 subunits of NF- $\mathrm{kB}$ to regulate their activity. For example, association of Bcl-3 with the p52 subunit strongly enhances the potential of p52 to activate transcription of an NF- $\mathrm{kB}$-dependent

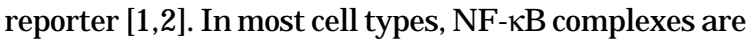
predominantly cytoplasmic and thus transcriptionally inactive until a cell is activated by a relevant stimulus.
In response to pro-inflammatory cytokines such as tumour necrosis factor (TNF) and interleukin (IL)-1, bacterial lipopolysaccharide (LPS), or a variety of other stimuli, I $\kappa \mathrm{B} \alpha$ and $\mathrm{I} \kappa \mathrm{B} \beta$ are phosphorylated on two serine residues located within the $\mathrm{N}$-terminal portion of the peptides $[1,2,4]$. This phosphorylation of $\mathrm{I} \kappa \mathrm{B}$ results in ubiquitination and subsequent degradation by the $26 \mathrm{~S}$ proteasome. A high-molecularweight complex (I $\mathrm{B}$ kinase, or IKK) responsible for phosphorylating $\mathrm{I} \kappa \mathrm{B} \alpha$ and $\mathrm{I} \kappa \mathrm{B} \beta$ was recently identified, which contains two key catalytic subunits, IKK $\alpha$ and IKK $\beta$ [reviewed in Ref. 4]. Degradation of the I $\mathrm{B}$ proteins results in the liberation of NF- $\kappa \mathrm{B}$ allowing translocation to the nucleus, where it can regulate the expression of specific genes typically involved in immune and inflammatory responses and in cell growth control [1,2].

\section{NF-kB and oncogenesis}

$\mathrm{v}$-Rel, a viral homologue of c-Rel, was identified as the transforming gene of an avian retrovirus which is highly oncogenic and causes aggressive tumors in chickens (reviewed in Refs [3,5]). More relevant to human cancer, the genes encoding c-Rel, NF-кB2 (p100/p52), and Bcl-3 are amplified and/or rearranged in certain cancers. For example, the $\mathrm{Bcl}$-3 gene was localized to a $[\mathrm{t}(14,19)$ (q32; 13.1$)]$ chromosomal translocation found in some B-cell chronic lymphocytic leukemias and is overexpressed in certain B-cell neoplasms [3,5]. Mutations in the gene encoding I $\mathrm{B} \alpha$ have been detected in Hodgkin's lymphoma [6] and are suggested to contribute to the constitutively active NF- $\kappa$ B in Hodgkin's cells. This implies a tumor suppressor function for $\mathrm{I} \kappa \mathrm{B}$ as well as an involvement of NF- $\kappa \mathrm{B}$ in this disease (see below).

Other evidence links NF- $\mathrm{kB}$ with oncogenic transformation. For example, the Tax oncoprotein from the human T-cell leukemia virus-I (HTLV-I) induces $\mathrm{NF}-\kappa \mathrm{B}$ activity, and activation of NF- $\mathrm{BB}$ was shown to be required for transformation of rat fibroblasts by the HTLV-I Tax protein [3]. Similarly, most viral transforming proteins stimulate NF- $\mathrm{BB}$ transcriptional activity at one level or another [7]. Consistent with a possible role in transformation and tumorigenesis, many human-derived, solid-tumor cell lines display increased nuclear NF- $\mathrm{KB}$ (p50-p65 heterodimer) levels and activity [3], although this does not always reflect the natural state of the tumor.

More evidence supports the involvement of NF- $\kappa \mathrm{B}$ in oncogenesis. First, it was shown that inhibition of NF- $\kappa \mathrm{B}$ by expression of a modified form of $\mathrm{I} \kappa \mathrm{B} \alpha$ (super-repressor $\mathrm{I} \kappa \mathrm{B} \alpha$ ) blocked focus formation induced by oncogenic H-Ras in NIH3T3 


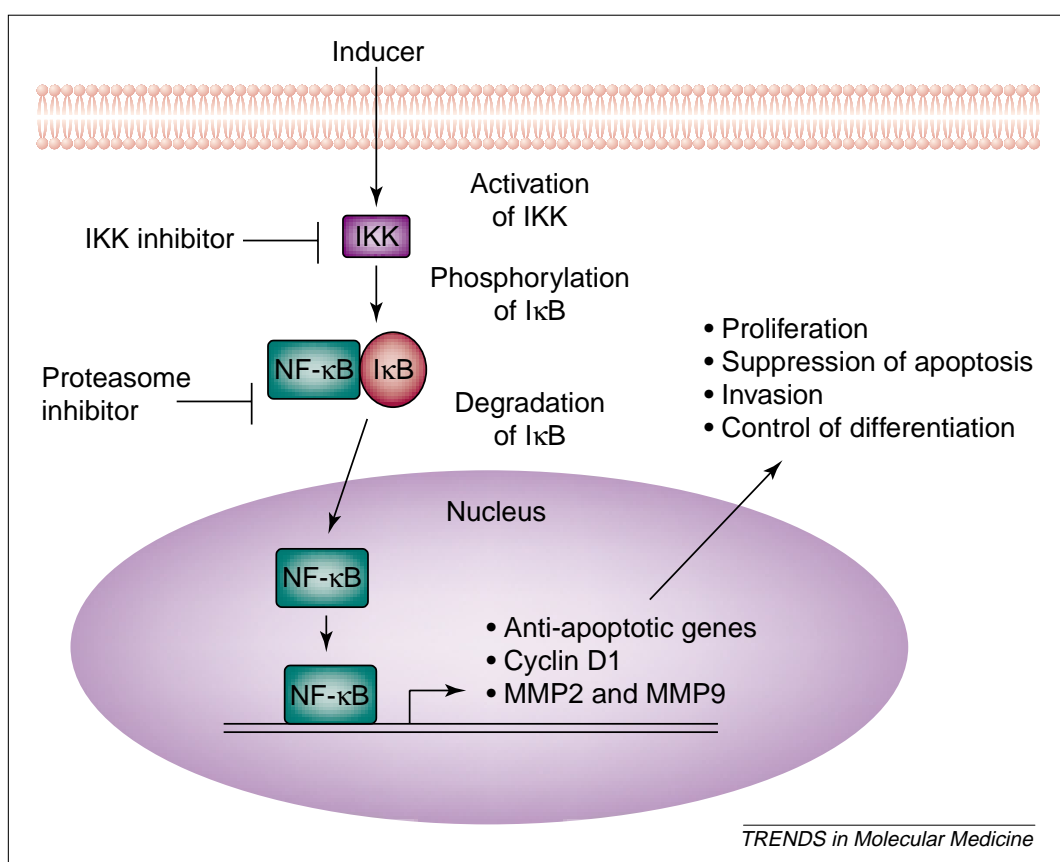

Fig. 1. Model for the activation of nuclear factor (NF)- $\mathrm{kB}$ by a variety of inducers and for the nuclear response controlled by NF- $\kappa B$. Inducers of NF- $\mathrm{KB}$ activate I $\mathrm{KB}$ kinase (IKK), which leads to phosphorylation of I $\mathrm{B}$ subunits on $\mathrm{N}$-terminal serines. Phosphorylated I $\kappa B$ is ubiquitinated and subsequently degraded by proteasome activity, allowing the accumulation of NF- $\kappa B$ in the nucleus. In the nucleus, NF-kB binds to regulatory regions in promoters and enhancers to stimulate transcription of genes associated with oncogenesis, suppression of apoptosis, and invasion. IKK inhibitors block $\mathrm{NF}-\mathrm{kB}$ activation through suppression of $1 \kappa B$ phosphorylation and proteasome inhibitors block $1 \kappa B$ degradation. cells (reviewed in Ref. [3]). Consistent with this finding, Ras-induced transformation is blocked by dominant-negative forms of IKK which block NF-кB [8]. Second, it was shown that the oncogenic fusion protein BCR-ABL associated with chronic myelogenous leukemia and some cases of acute lymphoblastic leukemia activated NF- $\mathrm{KB}$, and that tumorigenesis driven by BCR-ABL-expressing cells could be blocked by expression of the super-repressor $\mathrm{I} \kappa \mathrm{B} \alpha$ [3]. Additionally, NF- $\kappa \mathrm{B}$ was shown to be activated in Hodgkin's lymphoma, and inhibition of NF- $\mathrm{BB}$ blocked growth of these cells [9]. As mentioned earlier, there are numerous reports of NF- $\mathrm{NB}$ being activated in a variety of tumor cell lines grown in vitro [3]. For example, the classic form of NF- $\mathrm{NB}$ (p50-p65) has been shown to be activated (i.e. it has nuclear localization) in breast cancer cell lines and in some breast tumors [10]. Our observations suggest that NF- $\kappa$ B appears to be dysregulated in breast cancer, but we have found that human breast tumors more typically display an accumulation of nuclear p52 and Bcl-3 along with c-Rel rather than p65 [11]. Recently, it has been shown that inhibition of NF- $\mathrm{KB}$ in head and neck squamous cell carcinoma inhibits cell survival and tumor growth [12]. Consistent with the observations described above, NF- $\mathrm{kB}$ is activated by most, if not all, oncoproteins through signal transduction cascades that ultimately promote NF- $\mathrm{\kappa B}$ nuclear translocation or stimulate inherent transcriptional activity [3].

It should be noted that much of the evidence linking NF- $\mathrm{kB}$ with oncogenesis has been obtained using the so-called super-repressor form of $\mathrm{I} \kappa \mathrm{B} \alpha$ to

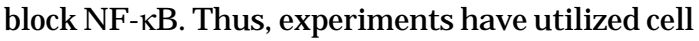
lines that stably express the modified, degradationresistant $\mathrm{I} \kappa \mathrm{B} \alpha$ or have utilized adenoviral delivery of the inhibitor [3]. Obviously, if I $\mathrm{B}$ can inhibit $\mathrm{NF}-\kappa \mathrm{B}$-independent pathways, then the proposed link between NF-kB and oncogenesis can be called is not specific for NF- $\mathrm{NB}$.

\section{Apparent roles for NF-kB in oncogenesis involve suppression of apoptosis, induction of proliferation, cell invasion and angiogenesis}

Numerous studies have indicated that NF- $\kappa \mathrm{B}$ activation can block cell-death pathways (reviewed in Ref. [3]). Thus, it was shown that NF- $\kappa B$ activation is required to protect cells from the apoptotic cascade induced by TNF and other stimuli. NF- $\kappa \mathrm{B}$ activates TRAF 1 and -2 and c-IAP1 and -2 to block potential TNF-induced caspase- 8 activity. Other anti-apoptotic genes have been shown to be activated by NF- $\mathrm{BB}$ and include the Bcl-2 homologs A1/Bfl-1, Bcl-xL, IEX-1, and XIAP. Of significant interest regarding the suppression of apoptosis are the observations that $\mathrm{NF}-\kappa \mathrm{B}$ can antagonize p53 function, possibly through the cross-competition for transcriptional co-activators [13]. However, other research groups have found a dependence on NF- $\mathrm{NB}$ for p53-dependent apoptosis [14]. The potential of NF- $\kappa \mathrm{B}$ to function as a pro-apoptotic factor is discussed below.

It has been proposed that suppression of apoptosis is associated with oncogenic potential. Inhibition of $\mathrm{NF}-\kappa \mathrm{B}$, via expression of the super-repressor $\mathrm{I} \kappa \mathrm{B} \alpha$, leads to the induction of apoptosis when an oncogenic allele of H-Ras (RasV12) is expressed (reviewed in Ref. [3]), suggesting that one role for NF- $\mathrm{kB}$ activation is to suppress transformation-associated apoptosis. Other evidence suggests that inhibition of NF- $\kappa$ B in transformed cells can induce apoptosis. Additionally, activation of NF- $\kappa \mathrm{B}$ by growth factors suppresses the apoptotic response induced by c-Myc expression during growth factor deprivation.

A further mechanism whereby NF- $\mathrm{BB}$ might be involved in oncogenesis is through the promotion of cell proliferation. NF- $\mathrm{KB}$ and Bcl-3 can promote cell growth through binding to the promoter of cyclin D1 and thus stimulating gene transcription (reviewed in Ref. [3]). Upregulation of cyclin D1 gene expression by NF- $\mathrm{kB}$ is associated with enhanced transition from $\mathrm{G}_{1}$ to $\mathrm{S}$ phase and retinoblastoma $(\mathrm{Rb})$ hyperphosphorylation (Fig.1).

What other NF- $\mathrm{BB}$-regulated genes or processes might contribute to oncogenesis? Cox-2, a protein involved in inflammation, is found upregulated in more aggressive forms of colorectal cancer, is known to be transcriptionally activated by NF- $\mathrm{KB}[1,2]$ and is known to promote angiogenesis [15]. Inhibition of $\mathrm{NF}-\kappa \mathrm{B}$ suppressed angiogenesis along with vascular endothelial growth factor (VEGF) and IL-8 expression in one model [16], but it is unclear whether this is an indirect response as smaller tumors caused by inhibition of NF- $\mathrm{KB}$ could be less hypoxic and therefore have less angiogenesis. Relative to other aspects of oncogenic control, NF- $\mathrm{\kappa B}$ is known to regulate expression of cell-adhesion molecules [1,2] and cell-surface protease (such as MMP-9 and MMP-2, see Ref. [3]). These observations are consistent with 
reports indicating that $\mathrm{NF}-\mathrm{\kappa B}$ can promote metastasis

$[17,18]$. Recently, it was shown that TNF-null animals are inhibited in their ability to undergo skin carcinogenesis [19]. As NF- $\mathrm{kB}$ positively regulates TNF gene expression and as TNF activates NF- $\kappa B$ [1,2], these results might correlate with a role for $\mathrm{NF}-\kappa \mathrm{B}$ in skin carcinogenesis. Consistent with the involvement of cytokines in cancer, it has been suggested that the upregulation of NF-kB in head and neck squamous cell carcinoma promotes pro-inflammatory cytokine production and possibly metastasis [20]. Similarly, $\mathrm{TNF} \alpha$ plays an important role in multiple myeloma through the NF-kB-dependent upregulation of IL-6 in bone marrow stromal cells [21]. IL-6 promotes growth and survival of multiple myeloma cells. More recently, NF- $\mathrm{BB}$ has been shown to block differentiation of myoblasts into myofibers through the induced post-transcriptional loss of MyoD mRNA. [22]. Thus, it is possible that NF- $\mathrm{kB}$ activation could promote loss of differentiation in certain tumors.

\section{Current approaches to inhibit NF-kB: concem about specificity?}

Original approaches to inhibit NF- $\mathrm{kB}$ included expression of the super-repressor form of $\mathrm{I} \kappa \mathrm{B} \alpha$ and the SN50 peptide, which blocks the nuclear localization of the p50 NF- $\mathrm{BB}$ subunit [3,23]. Recently, proteasome inhibitors have been used to block NF- $\mathrm{BB}$ nuclear localization through inhibition of $\mathrm{I} \kappa \mathrm{B} \alpha$ degradation and the processing of the $\mathrm{p} 105 \mathrm{NF}-\mathrm{\kappa B}$ precursor $[2,23,24]$. Several studies have shown that proteasome inhibitors have significant anti-tumor activity in murine xenograft models [24-26], and one of these, PS-341, inhibits NF- $\mathrm{kB}$ and, correspondingly, inhibits cell survival, tumor growth and angiogenesis in squamous cell carcinoma tumor models [27]. As the major proteolytic system in eukaryotic cells [28], however, the ubiquitin-proteasome pathway controls the breakdown of most cellular proteins, and thereby influences a variety of physiological processes. Obviously, a proteasome inhibitor will affect the stability of numerous proteins normally controlled by the ubiquitin-proteasome degradation pathway. Specificity therefore, regarding NF- $\mathrm{BB}$ as a unique target, is an issue with these types of agents.

Other molecules have been shown to inhibit $\mathrm{NF}-\kappa \mathrm{B}$, including a peptide that blocks the interactions of IKK $\gamma$ with the catalytic core of IKK, the non-steroidal anti-inflammatory drug (NSAID) sulindac, cyclopentenone prostaglandins, arsenic trioxide, thalidomide, a variety of anti-oxidants, natural products such as parthenolide and resveratrol, and pharmaceutically developed synthetic small-molecule inhibitors of the IKK complex $[23,29,30]$. With relevance to cancer, resveratrol was shown to induce apoptosis of Ras-transformed cells, and sulindac also induced cell death in certain transformed cells [3,23]. Arsenic trioxide, which directly modifies IKK on a critical cysteine, has shown efficacy in several hematological malignancies [29,31]. Recently, a drug (PS-1145) was identified that blocks I $\kappa$ B kinase (IKK) and has potent NF- $\mathrm{KB}$ inhibitory properties [32]. PS-1145 blocks multiple myeloma cell proliferation but not as effectively as the proteasome inhibitor PS-341 [32]. Overall, these data underscore the potential relevance of targeting NF- $\mathrm{KB}$ in cancer through monotherapies that block different constituents in the NF- $\kappa \mathrm{B}$ signal-transduction pathway.

\section{NF- $k B$ and chemo- and radio-resistance: is NF- $k B$ always anti-apoptotic?}

The realization that NF- $\kappa \mathrm{B}$ can inhibit apoptosis led us to examine whether or not NF- $\mathrm{KB}$ is induced by chemotherapy and whether it plays a role in blocking the efficacy of cancer chemotherapies and radiation.

We showed that HT1080 fibrosarcoma cells exposed to ionizing radiation or to the chemotherapy daunorubicin exhibited enhanced activation of NF- $\mathrm{KB}$ [3].

Inhibition of NF- $\mathrm{KB}$ under these experimental conditions led to a dramatically improved apoptotic response to ionizing radiation or daunorubicin treatment as compared with the control cells. HT1080 fibrosarcoma tumors grown in nude mice were induced to undergo apoptosis when infected with an adenovirus expressing a modified form of I $\mathrm{BB} \alpha$ along with systemic delivery of the chemotherapeutic agent CPT-11 [3]. Other tumors (e.g. those derived from the colorectal tumor cell line LOVO) showed basically identical responses to the combined treatment. In fact, LOVO tumors could be eliminated with CPT-11 systemic treatment and with adenoviral delivery of I $\mathrm{B} \alpha$ [3]. The use of the proteasome inhibitor PS-341 as a systemic inhibitor of NF- $\mathrm{\kappa B}$ in conjunction with CPT-11 treatment also led to significantly improved chemotherapeutic responses through enhanced apoptosis [33]. Lactacystin, a structurally unrelated proteasome inhibitor, has also been reported to enhance apoptosis induced by drugs such as etoposide and doxorubicin [3]. It should be noted that wild-type p53 is apparently not needed for the enhanced apoptotic response observed with chemotherapy plus $\mathrm{NF}-\kappa \mathrm{B}$ inhibition, given that the response occurs in cancer cells that have mutated or deleted p53 [33]. Similar to the chemotherapy data, inhibition of $\mathrm{NF}-\kappa \mathrm{B}$ sensitized LOVO cells to radiation with an approximately $80 \%$ reduction in tumor volume using xenografts receiving radiation and PS-341 [34].

The form of chemotherapy used might determine

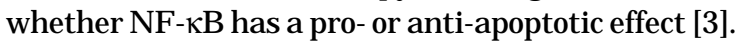
For example, the majority of chemotherapies that we have studied induce some form of DNA damage, and inhibition of NF- $\mathrm{KB}$ activation promotes cell death. However, it was proposed recently that NF- $\mathrm{B}$ might be required for Taxol-induced cell death [35]. This is consistent with studies that suggest that NF- $\mathrm{\kappa B}$ can function pro-apoptotically based on the type of stimulus to which cells are exposed. For example, cell death induced by hydrogen peroxide has been reported to require $\mathrm{NF}-\mathrm{\kappa B}$, and there is a report that 
$\mathrm{NF}-\kappa \mathrm{B}$ is required for p53-dependent cell death [14].

More studies are needed to determine chemotherapy specificity and potential tumor specificity of NF- $\kappa \mathrm{B}$ in controlling cancer therapy efficacy.

\section{Current clinical trial data relevant to the inhibition of NF-kB}

Several agents that are able to inhibit NF- $\mathrm{KB}$ function are currently in clinical use as cancer chemotherapeutics, whereas others are undergoing clinical development. One of the drugs in the former category is arsenic trioxide (ATO), whose use as Fowler's solution for the treatment of chronic myelogenous leukemia was first advocated in the 19 th century. More recently, ATO was reported to have activity against promyelocytic leukemia in studies from China [31]. A multicenter trial in the US in patients with relapsed acute promyelocytic leukemia (APL) found a complete response rate of 85\% [36], and ATO has been approved by the Food and Drug Administration for that indication. Studies have also found that ATO has comparable activity in patients with newly diagnosed APL [37]. ATO is being evaluated in ongoing clinical trials in patients with other hematological and solid tumors. Indeed, preliminary evidence of some activity in patients with multiple myeloma has recently been reported [36].

Thalidomide is another agent in clinical use that inhibits NF- $\mathrm{B}$, which it does at least partly by inhibiting I $\kappa \mathrm{B}$ kinase activity [30]. In patients with refractory multiple myeloma this drug has substantial activity, with a response rate of up to $32 \%$ [38]. Recent studies using combinations with other agents such as dexamethasone suggest that such regimens can have even greater efficacy (for example, see Ref. [39]). Interestingly, dexamethasone can inhibit NF- $\mathrm{KB}$ through mechanisms distinct from that of thalidomide [23]. These encouraging findings have prompted trials in other hematologic malignancies that have documented some activity in Hodgkin's and non-Hodgkin's lymphomas [40]. By contrast, while there is a response rate in AIDS-related Kaposi's sarcoma [41], the results with single agent thalidomide in other solid tumors have been more disappointing. Occasional responses have been noted in patients with gliomas [42] and androgen-independent prostate cancer [43], but of those patients who have derived any benefit most have exhibited stable disease rather than regression. As is the case in myeloma there is the possibility that combinations of thalidomide with standard chemotherapeutic agents may prove more efficacious, and such studies are currently underway (for example [44]).

While ATO and thalidomide were already in clinical use before their abilities to inhibit NF- $\mathrm{kB}$ were identified, several newer classes of chemotherapeutics are in development that were intended, at least in part, to target NF-кB. The best example of agents in this category are proteasome inhibitors, and specifically PS-341 [24]. Several Phase I studies of this drug have been completed, and excitement has been generated in the field of multiple myeloma research as significant activity was seen in that patient population including one complete response [45]. This prompted Phase II trials, which are currently underway [46]. In patients with solid tumors, the drug has been well-tolerated and an occasional response has been seen (for examples, see Refs [47,48]). Based on some of the preclinical data described earlier, combination Phase I trials of PS-341 with standard chemotherapy drugs such as CPT-11 and gemcitabine are ongoing, and if successful will lead to Phase II studies where their promise can be better evaluated. Given that proteasome inhibition also augments radiation sensitivity, clinical trials evaluating this approach are also underway.

With respect to the available clinical data, it is interesting to note that drugs that target NF- $\kappa$ B such as thalidomide and PS-341 have to date shown the greatest success as single agents in the therapy of hematological malignancies. This is perhaps reflective of the important role of NF- $\mathrm{KB}$ in the biology of diseases such as multiple myeloma where adherence of these cells to bone marrow stroma triggers synthesis of the myeloma survival and growth factor IL-6 through an NF- $\mathrm{BB}$-dependent mechanism [49]. The role of NF- $\mathrm{NB}$ in anti-apoptotic signaling has also raised new hopes that inhibitors will enhance the efficacy of conventional chemotherapeutic drugs. Because the NF- $\mathrm{kB}$ inhibitors in current clinical use might also work through other mechanisms (such as interruption of growth signaling pathways [50]), it will be difficult to determine how much of their activity is a result of their impact on NF- $\kappa \mathrm{B}$. The application of future agents such as IKK inhibitors that target the NF- $\mathrm{KB}$ signaling cascade more specifically will be more helpful in this regard. From a clinical perspective, a Phase III trial randomizing patients to, for example, either CPT-11 or CPT-11 and a specific NF- $\mathrm{KB}$ inhibitor would be required to prove that the preclinical xenograft data can be reproduced in a human population. Although such testing is likely to be several years away, at this time it appears to be one of the promising approaches to improving the clinical care and outcome of cancer patients worldwide.

\section{Concluding remarks}

There is significant enthusiasm from cell culture experiments and animal models for the use of NF- $\kappa \mathrm{B}$ inhibitors as a new anti-cancer therapy. However, it is presently unclear whether this type of approach will be of general applicability. Concerns range from the fact that current NF- $\mathrm{kB}$ inhibitors are not specific to issues regarding the potential pro-apoptotic and critical immunological functions of NF- $\mathrm{kB}$. Furthermore, another concern is the lack of knowledge about the specific roles of different NF- $\mathrm{kB}$ subunits, some of which might not be inhibited by drugs that block 
IKK activity or proteasome function. Moreover, the role of $\mathrm{NF}-\mathrm{\kappa B}$ could be different in different tumor types: for example, the function that NF- $\mathrm{KB}$ plays in leukemia and lymphoma might be different from that in solid tumor cells. Nevertheless, the current data are promising enough to strongly consider careful studies to dissect the roles of NF- $\mathrm{\kappa B}$ in a variety of cancers and to determine the applicability of inhibiting NF- $\mathrm{KB}$ as an adjuvant approach in standard approaches to cancer therapy.

\section{References}

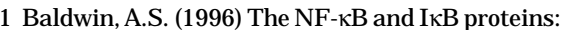
new discoveries and insights. Annu. Rev. Immunol. 14, 649-681

2 Ghosh, S. et al. (1998) NF- $\kappa B$ and Rel proteins: evolutionarily conserved mediators of the immune response. Annu. Rev. Immunol. 16, 225-260

3 Baldwin, A. (2001) Control of oncogenesis and cancer therapy resistance by the transcription factor NF-kB. J. Clin. Invest. 107, 241-246

4 Karin, M. and Ben-Neriah, Y. (2000) Phosphorylation meets ubiquitination: the control of NF- $\kappa \mathrm{B}$ activity. Annu. Rev. Immunol. 18, 621-663

5 Rayet, B. and Gelinas, C. (1999) Aberrant $\mathrm{Rel} / \mathrm{NF}-\kappa \mathrm{B}$ genes and activity in human cancer. Oncogene 18, 6938-6947

6 Cabannes, E. et al. (1999) Mutations in the I $\mathrm{B} \alpha$ gene in Hodgkin's disease suggest a tumor suppressor role for $\mathrm{I} \kappa \mathrm{B} \alpha$. Oncogene $18,3063-3070$

7 Mosialos, G. (1997) The role of Rel/NF-кB proteins in viral oncogenesis and the regulation of viral transcription. Semin. Cancer Biol. 8, 121-129

8 Arsura, M. et al. (2000) Role of the IкB kinase complex in oncogenic Ras and Raf-mediated transformation of rat liver epithelial cells. Mol. Cell. Biol. 20, 5381-5391

9 Bargou, R.et al. (1997) Constitutive NF-кB/RelA activation is required for proliferation and survival of Hodgkin's disease tumor cells. J. Clin. Invest. 100, 2961-2969

10 Sovak, M. et al. (1997) Aberrant NF-кB/Rel expression and the pathogenesis of breast cancer. J. Clin. Invest. 100, 2952-2960

11 Cogswell, P. et al. (2000) Selective activation of $\mathrm{NF}-\kappa \mathrm{B}$ subunits in human breast cancer:

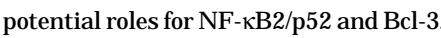
Oncogene 19, 1123-1131

12 Duffey, D. et al. (1999) Expression of a dominant mutant I $\mathrm{B} \alpha$ in human head and neck squamous cell carcinoma inhibits survival, proinflammatory cytokine expression, and tumor growth in vivo. Cancer Res. 59, 3468-3474

13 Webster, G. and Perkins, N. (1999) Transcriptional cross-talk between NF- $\mathrm{\kappa B}$ and p53. Mol. Cell. Biol. 19, 3485-3495

14 Ryan, K. et al. (2000) Role of NF-кB in p53-mediated programmed cell death. Nature 404, 892-897

15 Tsujii, M. et al. (1998) Cyclo-oxygenase regulates angiogenesis induced by colon carcinoma cells. Cell 93, 705-716

16 Huang, S. et al. (2000) NF- $\mathrm{NB}$ activity correlates with growth, angiogenesis and metastasis of human melanoma cells in nude mice. Clin. Cancer Res. 6, 2573-2581

17 Andela, V. et al. (2000) Tumor metastasis and the reciprocal regulation of prometastatic and antimetastatic factors by NF-кB. Cancer Res. $60,6557-6562$

18 Huang, S. et al. (2001) Blockade of NF- $\mathrm{kB}$ activity in human prostate cancer cells is associated with suppression of angiogenesis, invasion and metastasis. Oncogene 20, 4188-4197
19 Moore, R. (1999) Mice deficient in TNF $\alpha$ are resistant to skin carcinogenesis. Nat. Med. 5, 828-831

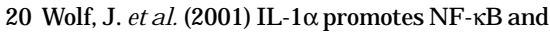
AP-1-induced IL-8 expression, cell survival and proliferation in head and neck squamous cell carcinomas. Clin. Cancer Res. 7, 1812-1820

21 Hideshima, T. et al. (2001) The role of TNF $\alpha$ in the pathophysiology of human multiple myeloma: therapeutic applications. Oncogene 20, 4519-4527

22 Guttridge, D. et al. (2000) NF-кB-induced loss of MyoD mRNA: possible role in muscle decay and cachexia. Science 289, 2363-2366

23 Yamamoto, Y. and Gaynor, R. (2001) Therapeutic potential of inhibition of the NF- $\mathrm{kB}$ pathway in the treatment of inflammation and cancer. J. Clin. Invest. 107, 135-142

24 Adams, J. (1999) Proteasome inhibitors: a novel class of potent and effective antitumor agents. Cancer Res. 59, 2615-2622

25 Orlowski, R.Z.et al. (1998) Tumor growth inhibition in a murine model of human Burkitt's lymphoma by a proteasome inhibitor. Cancer Res. $58,4342-4348$

26 Sun, J. (2001) CEP1612, a dipeptidyl proteasome inhibitor, induces p21WAF1 and p27KIP1 expression and apoptosis and inhibits the growth of the human lung adenocarcinoma A-549 in nude mice. Cancer Res. 61, 1280-1284

27 Sunwoo, J.et al. (2001) Novel proteasome inhibitor PS-341 inhibits activation of NF- $\mathrm{KB}$, cell survival, tumor growth, and angiogenesis in squamous cell carcinoma. Clin. Cancer Res. 7, 1419-1428

28 Orlowski, R.Z. (1999) The role of the ubiquitinproteasome pathway in apoptosis. Cell Death Differ. 6, 303-313

29 Kapahi, P. et al. (2000) Inhibition of NF- $\mathrm{kB}$ activation by arsenite through reaction with a critical cysteine in the activation loop of IKK. J. Biol. Chem. 275, 36062-36066

30 Keifer, J. et al. (2001) Inhibition of NF-kB activity by thalidomide through suppression of IкB kinase activity. J. Biol. Chem. 276, 22382-22387

31 Waxman, S. and Anderson, K. (2001) History of development of arsenic derivatives in cancer therapy. Oncologist 6(Suppl. 2), 3-10

32 Hideshima, T. et al. (2002) NF- $\mathrm{BB}$ as a therapeutic target in multiple myeloma. J. Biol. Chem. 277, 16639-16647

33 Cusack, J.et al. (2001) Enhanced chemosensitivity to CPT-11 with proteasome inhibitor PS-341: implications for systemic NF-кB inhibition. Cancer Res. 61, 3535-3540

34 Russo, S. et al. (2001) Enhancement of radiosensitivity by proteasome inhibition: implications for a role of NF-кB. Int. J. Radiat. Oncol. Biol. Phys. 50, 183-193

35 Huang, Y. et al. (2000) NF- $\mathrm{B} / \mathrm{I} \kappa \mathrm{B}$ signaling pathways may contribute to the mediation of paclitaxel-induced apoptosis in solid tumor cells. Cancer Res. 60, 4426-4432

36 Niu, C. et al. (1999) Studies on treatment of acute promyelocytic leukemia with arsenic trioxide: remission induction, follow-up, and molecular monitoring in 11 newly diagnosed and 47 relapsed acute promyelocytic leukemia patients. Blood 94, 3315-3324

37 Hussein, M.A. et al. 2001. A Phase II clinical study of arsenic trioxide (ATO) in patients (pts) with relapsed or refractory multiple myeloma (MM); a preliminary report. American Society of Hematology. Orlando, FL, pp. 378a.

38 Singhal, S. et al. (1999) Antitumor activity of thalidomide in refractory multiple myeloma. N. Engl. J. Med. 341, 1565-1571

39 Rajkumar, S.V.et al. 2001. Combination therapy with thalidomide plus dexamethasone (Thal / Dex) for newly diagnosed myeloma (MM), American Society of Hematology. Orlando, FL, pp 849a.

40 Pro, B. et al. 2001. Phase II study of thalidomide in patients with recurrent Hodgkin's disease (HD) and non-Hodgkin's lymphomas (NHL), American Society of Hematology. Orlando, FL, pp 246b.

41 Little, R.F. et al. (2000) Activity of thalidomide in AIDS-related Kaposi's sarcoma. J. Clin. Oncol. 18, 2593-2602

42 Fine, H.A. et al. (2000) Phase II trial of the antiangiogenic agent thalidomide in patients with recurrent high-grade gliomas. J. Clin. Oncol. $18,708-715$

43 Figg, W.D. et al. (2001) A randomized phase II trial of thalidomide, an angiogenesis inhibitor, in patients with androgen-independent prostate cancer. Clin. Cancer Res. 7, 1888-1893

44 Figg, W.D. et al. (2001) A randomized phase II trial of docetaxel (taxotere) plus thalidomide in androgen-independent prostate cancer. Semin. Oncol. 28, 62-66

45 Stinchcombe, T.E. et al. 2000. PS-341 is active in multiple myeloma: Preliminary report of a Phase I trial of the proteasome inhibitor PS-341 in patients with hematologic malignancies, American Society of Hematology. San Francisco, CA, pp 516a.

46 Richardson, P.G. et al. 2001. Phase II study of PS-341, a novel proteasome inhibitor, alone or in combination with dexamethasone in patients with multiple myeloma who have relapsed following front-line therapy and are refractory to their most recent therapy, American Society of Hematology. Orlando, FL, pp 774a.

47 Hamilton, A.L.et al. 2001. PS-341: Phase I study of a novel proteasome inhibitor with pharmacodynamic endpoints, American Society of Clinical Oncology. San Francisco, CA, pp 85a.

48 Aghajanian, C. et al. 2001. A Phase I trial of the novel proteasome inhibitor PS-341 in advanced solid tumor malignancies, American Society of Clinical Oncology. San Francisco, CA, pp 85a.

49 Chauhan, D. et al. (1996) Multiple myeloma cell adhesion-induced interleukin- 6 expression in bone marrow stromal cells involves activation of NF-kappa B. Blood 87, 1104-1112

50 Hideshima, T. et al. (2001) The proteasome inhibitor PS-341 inhibits growth, induces apoptosis, and overcomes drug resistance in human multiple myeloma cells. Cancer Res. 61, 3071-3076 